\title{
Rotor Losses in a Switched Reluctance Motor - Analysis and Reduction Methods
}

\author{
B. Schweighofer ${ }^{1, \text { a }}$, M. Recheis ${ }^{1}$, P. Fulmek ${ }^{2}$, and H. Wegleiter ${ }^{1}$ \\ 1 Institute of Electrical Measurement and Measurement Signal Processing, Graz University of Technology, Graz, Austria \\ 2 Institute of Sensor and Actuator Systems, Vienna University of Technology, Vienna, Austria
}

\begin{abstract}
Due to the increasing hybridization and electrification of vehicles, flywheel energy storage devices are an important area of research. In automotive application besides the weight criteria, some additionally constrains, such as size, efficiency and especially cost have to be fulfilled. Therefore typically a compact design, in which the rotor of the needed electrical machine simultaneously acts as storage mass is chosen. Since the machine is running in vacuum and the rotor can dissipate its heat only by means of thermal radiation or through the bearings if conventional bearings are used, the rotor losses play a vital role. In this work the rotor losses of a switched reluctance machine are analyzed in detail and a method to reduce the rotor losses is proposed.
\end{abstract}

\section{Introduction}

Due to the increasing hybridization and electrification of vehicles, flywheel energy storage devices are an important area of research. Popular choices for the required electrical machine are the permanent magnet $[3,4]$ or synchronous reluctance machine. Reluctance machines generally have the advantage that there are no electromagnetic spinning losses at zero torque, and the rotor can be constructed entirely from (high strength) electrical steel allowing a higher rotational speed. Using a switched reluctance machine instead of a synchronous one has additional benefits by means of increased power density and simpler windings at the cost of higher rotor losses. But those losses represent a big issue since the machine is running in vacuum and the rotor can dissipate its heat only by means of thermal radiation and through the bearings if conventional bearings are used.

This paper will deal with the analysis of the rotor losses of a switched reluctance machine and possibilities to reduce them. For this purpose the magnetic field of the motor is simulated with a FEM software resulting in a detailed description of the rotor losses and their distribution. The possible influence of the converter (especially due to the ripple current) is investigated. Also a specific pulse pattern is analyzed, whereby every pole in the rotor is always magnetized only in the same direction as the previous magnetization. Experimental measurements will be carried out to measure the actual reduction of the rotor warming.

\section{Simulation / Calculation}

Since the power loss depends on the drive pattern one has to calculate the magnetic field in the machine for every different drive pattern and motor speed. Instead of doing every calculation with a complete FEM analysis, we decided to calculate the machine losses by means of postprocessing pre-calculated maps. Even so the calculation

\footnotetext{
a e-mail: bernhard.schweighofer@tugraz.at
}

of the maps need some time the post-processing step itself just takes a few seconds. Testing many different drive patterns in a short amount of time allows e.g. to simultaneously optimize the torque ripple of the machine and the power loss inside the rotor for a given working point in a reasonable amount of time.

\subsection{Magnetic Field}

Starting with the 10/8 short flux path machine from [1] the design of the rotor was mechanically optimized to allow for high rotational speeds. The end result is shown in figure 1. Especially the problem with the stress in the area of a central shaft which would occur in all rapidly rotating machines has been solved by replacing the central shaft with bolts around the circumference. These bolts are mounted to high strength steel discs on both ends of the rotor stack. The discs serve a dual purpose. Firstly they have a stub shaft to mount mechanical bearings. To the other they can be used to increase the energy content of the flywheel by means of increasing the thickness or even the diameter of the discs. It is reasonable to use sheet metal for a large part of this additional flywheel mass instead of a solid block to reduce possible eddy current losses at the edge of the electrical machine. This is also recommended for security reasons in case of failure. With a bore hole diameter of $100 \mathrm{~mm}$ and a total rotor stack length of $120 \mathrm{~mm}$ the designed flywheel can store $100 \mathrm{Wh}$ at a speed of $60000 \mathrm{rpm}$.

The rotor is made of VACODUR 50 from the company Vacuumschmelze (high mechanical strength electrical steel) whereas the stator is made of conventional electrical steel (ARMCO 100). With an FE program ${ }^{1}$ the magnetic field has been calculated for various positions and current densities :

- from $0^{\circ}$ (aligned) to $25^{\circ}$ in one degree steps, so it covers slightly more than the unaligned position at $18^{\circ}$

\footnotetext{
${ }^{1}$ COMSOL Multiphysics, www.comsol.com
} 
- from 1 to $20 \mathrm{~A} / \mathrm{mm}^{2}$ in steps of $1 \mathrm{~A} / \mathrm{mm}^{2}$, additional points at $0.001,0.01,0.1,0.2,0.3$, and $0.5 \mathrm{~A} / \mathrm{mm}^{2}$

In total $26 \cdot 26=676$ calculations have been performed (which took about twelve hours on a fast desktop computer). As a result the FE program delivers the magnetic field inside the machine (figure 1), the magnetic flux $\phi$ (measured in the middle of a stator coil, figure 2) and the generated torque as a function from rotor position and coil current.

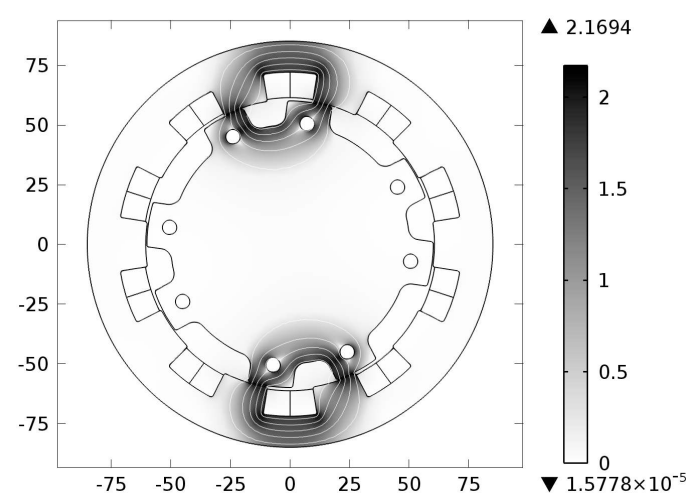

Figure 1. Calculated flux density $B$ at $7 \mathrm{~A} / \mathrm{mm}^{2}$. The rotor is moved $10^{\circ}$ from the aligned position.

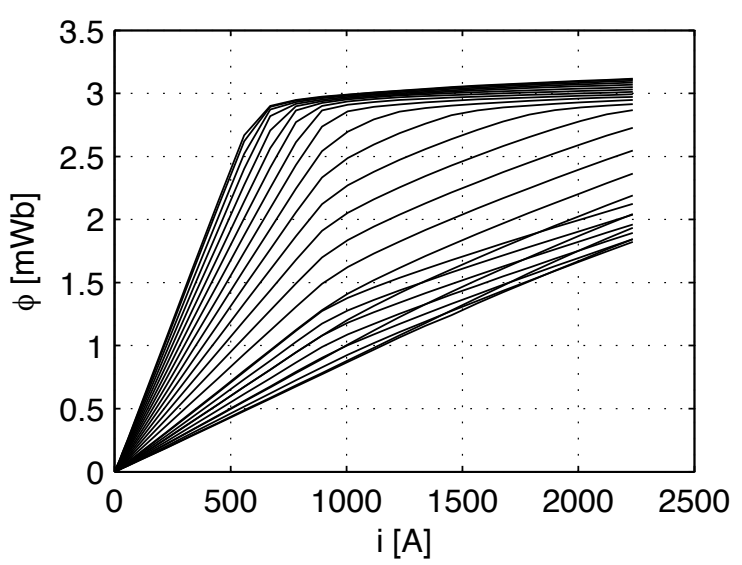

Figure 2. Magnetic flux $\phi$ as a function of the current $i$ and the rotor position $\alpha$ - from top (aligned) to bottom (unaligned).

\subsection{Current and Torque profile}

Since the magnetic flux is a function of the current $i$ inside the stator coil and the rotor position $\alpha$ the coil voltage is given by:

$$
V=i \cdot R+N \frac{\mathrm{d} \phi(i, \alpha)}{\mathrm{d} t}=i \cdot R+N \frac{\mathrm{d} \phi}{\mathrm{d} i} \frac{\mathrm{d} i}{\mathrm{~d} t}+N \frac{\mathrm{d} \phi}{\mathrm{d} \alpha} \frac{\mathrm{d} \alpha}{\mathrm{d} t}
$$

With the speed of the rotor $\omega=\mathrm{d} \alpha / \mathrm{d} t$ equation 1 can be rewritten as:

$$
\frac{\mathrm{d} i}{\mathrm{~d} t}=\frac{V-i \cdot R-N \frac{\mathrm{d} \phi}{\mathrm{d} \alpha} \omega}{N \frac{\mathrm{d} \phi}{\mathrm{d} i}}
$$

The current profile over time can now be calculated by means of numerical integration. The derivation for $d \phi / \mathrm{d} \alpha$ and $d \phi / \mathrm{d} i$ are calculated from the results of the FE simulation (figure 2). For the presented work the current was controlled by a simple on-off controller (for the driving voltage) in which turn on angle, turn off angle, peak current and current hysteresis where adjusted to get an output power of $20 \mathrm{~kW}$ for each of the simulations (figure 3 ). The torque of the machine is computed by means of a table lookup in the torque data indexed by the actual rotor position and current. In combination with the actual rotor speed also the mechanical power of the machine can be calculated. Figure 4 and 5 show some results of this calculation for $N=5$.

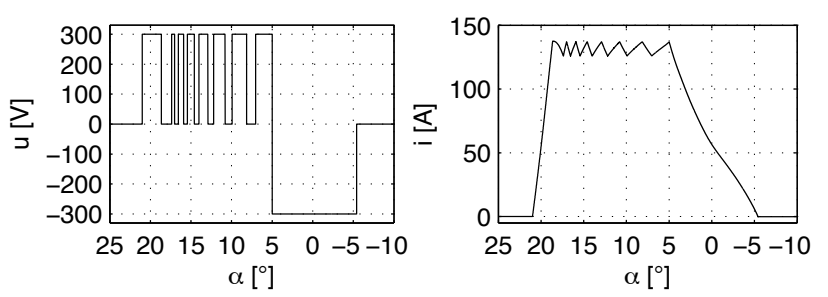

Figure 3. Voltage $u$ and current profile $i$ of a single stator coil with $N=5$ over rotor angle $\alpha$ at $20000 \mathrm{rpm}$.

\subsection{Losses}

In the further analysis we will concentrate on rotor losses, since they represent the bottleneck for the operation of the machine.

In principle, one must distinguish between two types of losses - eddy current and hysteresis loss.

\section{Eddy Current Losses / Skin Effect:}

In a first step the skin effect is calculated since it could significantly reduce the penetration depth and therefore the effective thickness of the laminations. From the data sheet of the VACODUR 50 material (variant with optimum mechanical properties) one gets the electrical resistivity $\rho=$ $0.42 \cdot 10^{-6} \Omega \cdot \mathrm{m}$ and the maximum value for the magnetic permeability $\mu_{\max }=0.0088 \frac{\mathrm{Vs}}{\mathrm{Am}}$. Since it takes 20 steps for a full revolution and each rotor tooth is active every second pulse the rotor tooth frequency is ten times higher than the mechanical speed. For a speed range of 20000 to $60000 \mathrm{rpm}$ one gets a tooth frequency in the range of $f_{\text {tooth }}=3.33 \div 10 \mathrm{kHz}$. This results in a minimum skin depth of

$$
\delta_{\min }=\sqrt{\frac{\rho}{\pi f \mu_{\max }}}=68 \div 39 \mu \mathrm{m}
$$

For the used lamination thickness of $100 \mu \mathrm{m}$ this effect can therefore be neglected in a first approximation. This also means that the static calculation in chapter 2.1 is valid and does not need any correction.

Since this material is designed with the aim of high mechanical strength the magnetic properties are not as good as with standard electrical sheets. The coercivity is much higher (in the range of $150 \div 200 \mathrm{~A} / \mathrm{m}$ ) leading to high hysteresis losses which dominate the total losses up to about 

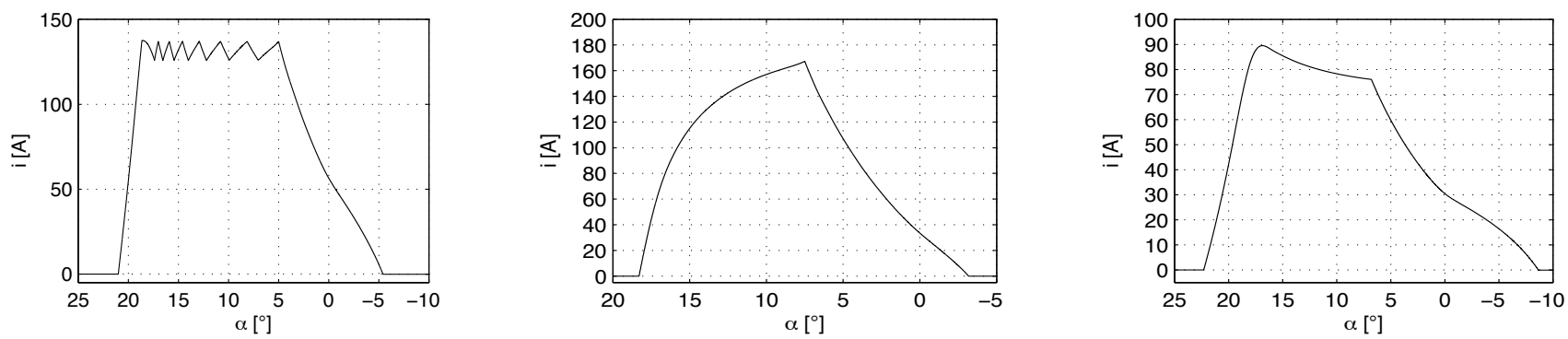

Figure 4. Current profile of a single stator coil over rotor angle $\alpha$. From left to right: $20000 \mathrm{rpm}$ with low ripple current, $20000 \mathrm{rpm}$ with a single voltage on/off pulse (and correspondingly higher ripple current), $60000 \mathrm{rpm}$ with a single on/off pulse.
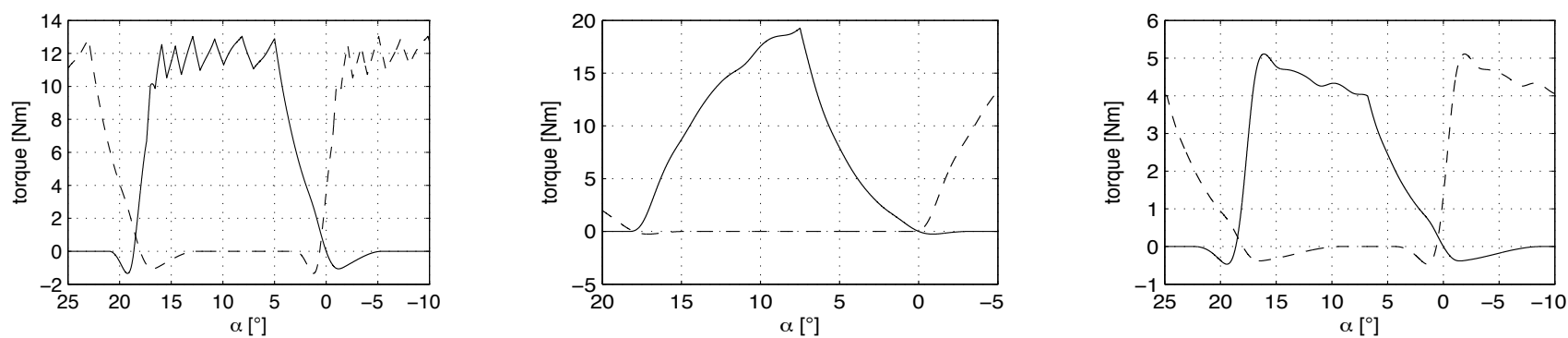

Figure 5. Torque profile over rotor angle $\alpha$. From left to right: $20000 \mathrm{rpm}$ with low ripple current, $20000 \mathrm{rpm}$ with a single on/off pulse, $60000 \mathrm{rpm}$ with a single on/off pulse. The dashed line additionally shows the torque of the previous and next stroke.

$1 \mathrm{kHz}$ (which is also the highest frequency shown in the data sheet). Hence, in a first approximation, the eddy current losses are neglected and only the hysteresis losses are considered.

\section{Hysteresis Losses}

The hysteresis loss can be calculated from the hysteresis loop (BH-loop). The needed flux density data $B$ over time can be calculated by means of a table lookup in the stored magnetic field data from the FE simulation (chapter 2.1) indexed by the current profile (course of current and rotor angle over time) calculated in the previous section. Since the used FE program does not calculate a hysteresis curve itself (only a static BH-curve is used), the missing data for the magnetic field $H$ has to be calculated in a different way. For this purpose the method described in [5] is used to calculate the magnetic field $H$ from the known flux density profile. In figure 6 the $\mathrm{BH}-$ loops for two regions in the rotor are shown.

Typically the controller is only able to drive the current in one direction (unipolar operation). For one stroke two adjacent stator coils have to be activated. In order to achieve the low flux operation adjacent stator coils have to be wound in opposite directions. Therefore a single rotor tooth always sees an alternating magnetic field from one stroke to another. Hence for a complete BH-loop in figure 6 it takes two strokes at the rotor tooth.

Using a more complex controller which allows to drive the coil current into both directions (bipolar operation) one can modify the switching pattern so that a single rotor tooth is always magnetized only into the same direction (figure 7). Now in every stroke a complete BH-loop is performed. So one has to take twice the area to compare it with the $\mathrm{BH}$-loops shown in figure 6. Integrating the losses over the complete rotor shows that with this method the rotor losses can be reduced by almost $40 \%$ in this operating point (from $463 \mathrm{~W}$ with the unipolar controller to $286 \mathrm{~W}$ with the bipolar controller). Now of course the sta- tor teeth see an alternating magnetic field and the losses in the stator will increase. But since the stator is made of a good electrical sheet (it does not need the high mechanical strength as in the rotor) the increase in power loss will be much lower than the decrease of losses inside the rotor. In sum this method will even decrease the total loss of the machine.

Going down to lower rotational speeds allows more freedom choosing current amplitude and hysteresis (e.g. left and middle plot in figure 4). Calculating the BH-loops and power loss inside the rotor (figure 8) shows that the loss for the low ripple version $(330 \mathrm{~W})$ is almost the same as for the low chopper frequency version $(327 \mathrm{~W})$. Therefore, in relation to power loss one can freely choose between low switching frequency or a reduced torque ripple.

\section{Experimental}

The previously shown machine is currently in production and will be available only towards the end of the year. In the meantime we have tested the different drive patterns with a commercially available SRM from the company "Motion System Tech Tokyo Japan". The RA165157 is an $8 / 6 \mathrm{SRM}$ with a rated power of $1.13 \mathrm{~kW}$ at a rated speed of $6000 \mathrm{rpm}$ (max. $12000 \mathrm{rpm})$. For the measurements a low speed (1300 rpm) is chosen, so eddy current losses can be neglected. Both tests were performed at a mechanical load of $2 \mathrm{Nm}$ (rated torque). As expected, the losses in the rotor are significantly lower for the bipolar drive pattern (figure 9, left, dashed). But a comparison with the previously performed calculation is not possible because the type of electrical steel in the machine is not known.

Furthermore, it is interesting to note that also the stator temperature settles down to a smaller value. This is attributed to the fact that the bulk of the losses in the machine 

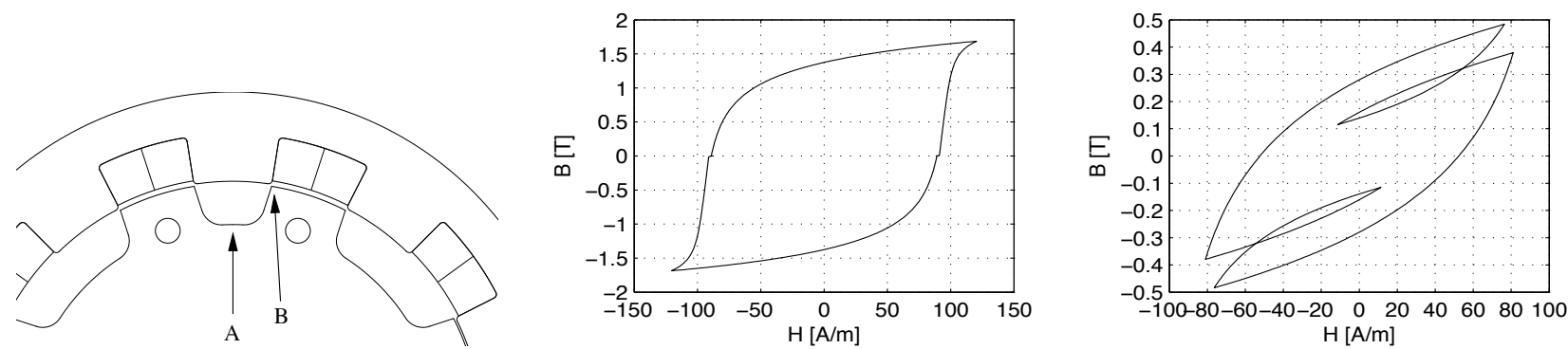

Figure 6. BH-loop (magnetic hysteresis) at $60000 \mathrm{rpm}$ at rotor position "A" (middle plot) and "B" (right plot).
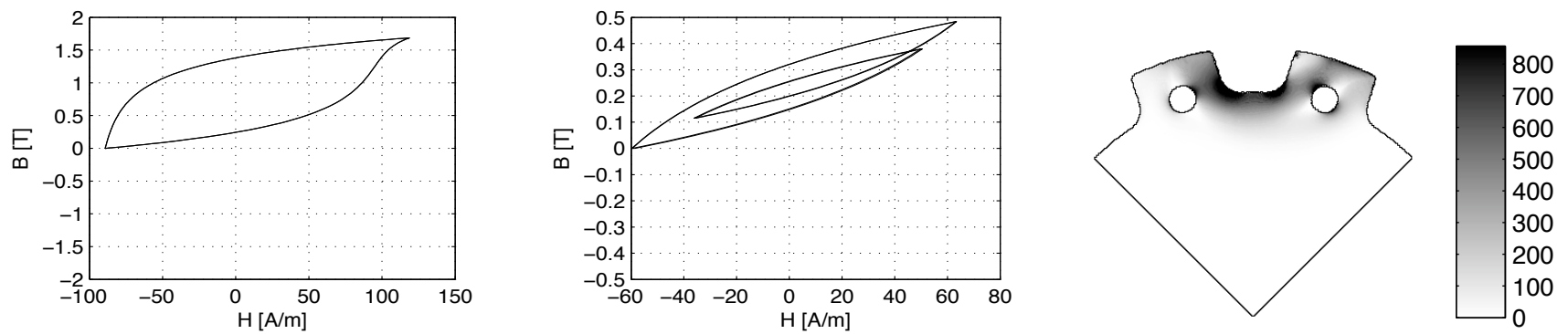

Figure 7. BH-loop at $60000 \mathrm{rpm}$ at rotor position "A" (left) and "B" (middle) for unipolar modulation of the rotor tooth. Additionally the power loss distribution inside the rotor is shown (right).
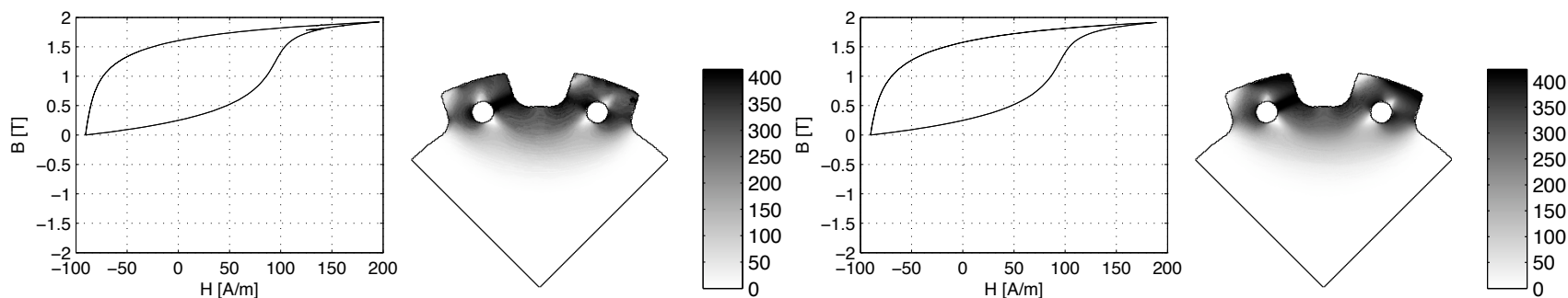

Figure 8. BH-loop (rotor position "A") and power loss distribution at $20000 \mathrm{rpm}$. Left - for the low ripple current version, right - for the single on/off version.

arise from the rotor, which can also be seen at the low temperature difference between the rotor and stator.
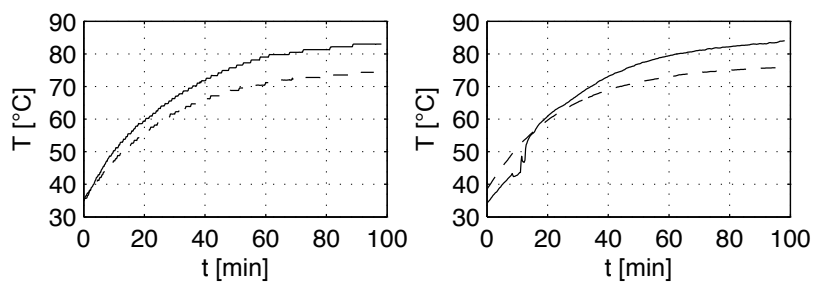

Figure 9. Heating curve for the rotor (left) and the stator (right). Solid - conventional drive pattern, dashed - special drive pattern. The disturbance at the beginning of the stator temperature measurements (solid curve to the right) is due to a loosely attached temperature sensor, which has been fixed at about $t=10 \mathrm{~min}$.

\section{Conclusion}

It could be shown that by using a special control of the switched reluctance machine the losses in the rotor can be reduced significantly. Disadvantages are the higher losses in the stator (which can be easily removed by cooling) and the somewhat greater expense for the inverter. Should the losses in combination with the duty cycle still be too high, so that the rotor heats up to much in a given application, a machine type with even lower rotor losses has to be chosen (e.g. synchronous reluctance machine).

\section{Acknowledgment}

This research has been supported be the "Neue Energien 2020" program of the "Klima- und Energiefonds" (Grant No. FFG 825553).

\section{References}

1. T. J. E. Miller, Switched Reluctance Motors and their Control (Magna Physics Publishing and Oxford Science Publications, 1993)

2. R. Krishnan, Switched reluctance motor drives: modeling, simulation, analysis, design, and applications (CRC Press LLC, 2001)

3. Z. Q. Zhu, D. Howe, IEE Proc.-Electr. Power Appl. 148(4), 299-308 (2001)

4. D. Johnson, P. Pillay, M. Malengret, IEEE Ind. Applic. Soc. 1, 57-63 (2001)

5. P. Fulmek, P. Haumer, H. Wegleiter, B. Schweighofer, COMPEL 29(6), 1504-1513 (2010) 